\title{
Perspective
}

PERSPECTIVE Actualité en histoire de l'art

1 | 2006

Antiquité/Moyen Âge

\section{Meyer Schapiro et la sculpture romane. Questions autour d'une non-réception en France}

Enrico Castelnuovo, Roland Recht et Robert A. Maxwell

\section{OpenEdition}

1 Journals

Édition électronique

URL : http://journals.openedition.org/perspective/4148

DOI : 10.4000/perspective.4148

ISSN : 2269-7721

Éditeur

Institut national d'histoire de l'art

Édition imprimée

Date de publication : 31 mars 2006

Pagination : 80-96

ISSN : 1777-7852

\section{Référence électronique}

Enrico Castelnuovo, Roland Recht et Robert A. Maxwell, « Meyer Schapiro et la sculpture romane.

Questions autour d'une non-réception en France », Perspective [En ligne], 1 | 2006, mis en ligne le 31 mars 2018, consulté le 01 octobre 2020. URL : http://journals.openedition.org/perspective/4148 ;

DOI : https://doi.org/10.4000/perspective.4148 


\section{Meyer Schapiro et la sculpture romane. Questions autour d'une non-réception en France}

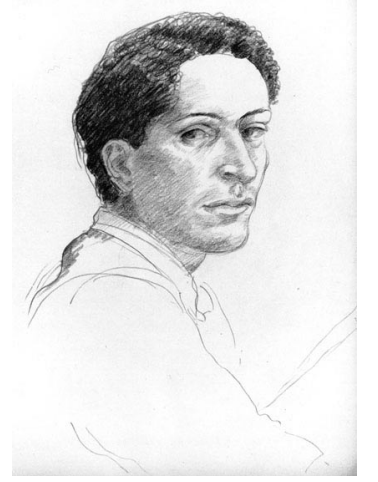

Autoportrait, Meyer Schapiro, 1931.

Meyer Schapiro (Siauliai, Lithuanie, 1904-Greenwich Village, États-Unis, 1996), enseignant à Columbia University depuis 1928, a été I'une des figures marquantes du milieu intellectuel new-yorkais dès 1930, aussi par son engagement politique et son soutien à l'expressionnisme abstrait américain ${ }^{1}$. Après une thèse sur le décor sculpté de Moissac soutenue en 1929, il s'intéressa aussi bien à l'art roman qu'à l'art des XIX ${ }^{\mathrm{e}}$ et XX $\mathrm{X}^{\mathrm{e}}$ siècles, et à la théorie de l'art dans une démarche qui scrute "I'intégrité formelle de I'objet esthétique » (Michael Ann Holly). L'ensemble de ses écrits a été publié sous forme de recueils, dont un sur l'art roman, et un autre intitulé Theory and Philosophy of Art: Style, Artist, and Society, (New York, 1994), qui a donné lieu à de nombreux comptes rendus. C'est en effet cet aspect de l'œuvre intellectuelle de Schapiro qui a été surtout glosé ${ }^{2}$ ou diffusé, alors que ses études très novatrices sur la sculpture romane et l'esthétique médiévale peinent à être connues en Europe, malgré d'intéressantes mises en perspectives ${ }^{3}$.

\author{
Débat entre Enrico Castelnuovo, Roland Recht et Robert A. Maxwell
}

Pour mieux comprendre la méthode et la pensée de Meyer Schapiro sur I'art roman et le problème de sa non-réception en France, Perspective a interrogé trois historiens de l'art du Moyen Âge, de génération et de tradition historiographique différentes : Enrico Castelnuovo, professeur émérite à la Scuola Normale Superiore di Pisa, connu pour ses travaux sur l'art médiéval et ses recherches sur art et société ; Roland Recht, professeur au Collège de France, spécialiste des chantiers médiévaux et des questions du patrimoine comme de l'histoire de l'histoire de l'art ; Robert A. Maxwell, Assistant Professor à la University of Pennsylvania, qui étudie l'art roman et son historiographie.

Perspective. La plus grande partie des écrits de Meyer Schapiro sur la sculpture romane (Moissac, 1931 ; Souillac, 1939; Silos, 1939) se concentre dans la décennie 1930. Ils sont certes liés à sa thèse sur la sculpture à Moissac, soutenue en 1929 à Columbia University, mais par la suite Schapiro publia plutôt sur des problèmes plus actuels et plus proches de l'art contemporain, de Courbet et l'imagerie populaire à l'interprétation proposée par Freud d'un tableau de Léonard de Vinci, sans oublier ses réflexions sur la notion de style. Quelle fut la place des études sur la sculpture romane dans l'évolution de la pensée de Meyer Schapiro? Comment expliquer, après 1945, ce relatif désintérêt, même si la méthode de travail de Meyer Schapiro était celle d'un vagabondage (au sens noble) intellectuel?

Robert Maxwell. Avant d'aborder la place de la sculpture romane dans la pensée de Schapiro, permettez-moi de repenser la question de sa bio-bibliographie autrement. L'art roman n'a jamais quitté sa pensée, de la même façon que l'art moderne n'y était jamais absent non plus. Vouloir distinguer des phases dans sa pensée selon ce type de schéma serait infructueux. Rappelons-nous que son essai sur I'« attitude esthétique » à l'époque romane est paru en $1947^{4}$, celui sur des dessins d'Auxerre en $1954{ }^{5}$ et celui sur un relief à Rodez en $1963^{6}$, et son unique livre (à proprement parler, puisque certains essais conçus pour des revues sont sortis en livre) est celui sur un manuscrit roman, paru en $1964^{7}$. Appelé à participer aux Charles Eliot Norton Lectures à Harvard en $1967^{8}$, Schapiro les a consacrées à la sculpture monumentale et architecturale à l'époque romane. L'art roman, par ailleurs, prend une place majeure dans ses deux écrits importants sur la sémiotique ${ }^{9}$, dont la conception et l'argumentation sont tout à fait nourries de ses réflexions sur l'art des $\mathrm{XI}^{\mathrm{e}}$ et $\mathrm{XI}{ }^{\mathrm{e}}$ siècles. Son ultime projet, édité seulement en $2005{ }^{10}$, portait sur l'enluminure anglo-saxonne, et si ce sujet ne porte pas directement sur l'art roman, il montre bien que l'intérêt pour l'art de cette période ne s'est jamais complètement effacé.

Il est à noter également que la majorité de ses écrits publiés pendant sa période « romane » (1931-35) traitent de l'art moderne. C'est pourquoi il serait inopérant à mon 
sens d'en faire deux Schapiro, l'un médiéviste et l'autre moderniste. Sa pensée sur l'art roman et sur l'art moderne, qui a mûri au fil des années, se faisait en tandem, sa réflexion sur l'un nourrissant l'autre. II rapprochait rarement des œuvres des deux époques (mis à part dans ses essais sur la sémiotique ou en se référant à un exemple précis dans une note de bas de page), préférant faire dialoguer des considérations et réflexions inspirées de chacune d'elles. Bien évidemment, certains de ses travaux, comme son essai sur " l'attitude esthétique » à l'époque romane, sont trempés d'une pensée moderniste, mais plus qu'un regard contemporain, il opère dans ses œuvres une dialectique entre une esthétique empreinte des enjeux sociaux de son époque et les créations du passé : il s'agit là toujours d'une recherche herméneutique et évolutive.

Aborder ainsi son œuvre sur l'art roman, c'est aussi s'approcher de Schapiro le moderniste. Et inversement, étudier des essais comme Les pommes de Cézanne ${ }^{11}$ ou $L^{\prime}$ art abstrait ${ }^{12}$ sans lire ses études sur Léonard ${ }^{13}$ ou la sculpture romane ne saurait que donner une lecture partiale, voire déformée de son travail. Isoler l'un de ses écrits risquerait de le figer dans son temps sans tirer les leçons d'une méthode toujours en formation.

Enrico Castelnuovo. On pourrait discuter pour savoir si ce désintérêt est réel. Si l'essai "On the aesthetic attitude in Romanesque Art », qui est extrêmement important, remonte à 1947, celui sur le relief de Rodez, lui aussi capital, est de $1963^{14}$. De plus, ce dernier n'est pas un écrit d'« occasion » sorti d'un vieux tiroir pour des Mélanges, mais une communication rédigée pour un congrès international pour lequel il aurait pu choisir un sujet « moderne ». Il y est question d'un relief qu'il avait vu quand il était étudiant en 1926, on pourrait donc le considérer comme un sous-produit de l'étude sur Moissac; mais ce n'est pas le cas, car la réflexion sur les origines de la sculpture romane en France, et par là sur la date des chapiteaux de Cluny, est à l'époque nouvelle et fort importante. D'autre part ce ne sont pas un mais deux des cinq volumes de ses œuvres complètes ${ }^{15}$ que Meyer Schapiro a consacrés au Moyen Âge dans le sens le plus vaste du terme. Car Late, Antique, Early Christian and Mediaeval Art (1979) rentre aussi dans le sujet. Il est d'autre part difficile de séparer la réflexion sur la sculpture de celle sur l'enluminure ou la peinture. Un texte comme The Parma Ildefonsus (1964) prend en compte également les styles de la peinture et de la sculpture à Cluny autour de 1110.

Roland Recht. Je ne parlerai pas de "désintérêt » pour l'art roman après 1945 : Meyer Schapiro publie plusieurs articles très importants sur l'art pré-roman, paléo-chrétien et byzantin durant la décennie 1950. Ce sont alors avant tout des études d'iconographie religieuse. L'étude iconographique a été l'un des centres d'intérêt de Meyer Schapiro depuis sa thèse sur Moissac. Et dans ce sens, ses travaux sur le XIX ${ }^{\mathrm{e}}$ siècle sont restés en partie fidèles à ses préoccupations de toujours. L'autre grand centre d'intérêt est l'étude matérielle des œuvres. J'y reviendrai plus loin.

Perspective. Comment s'articulaient, dans les années 1930, les travaux sur la sculpture romane de Meyer Schapiro, au sein des études américaines de Arthur Kingsley Porter, des essais d'Émile Mâle et de Henri Focillon, des réflexions de Jurgis Baltrusaïtis (Schapiro publie un compte rendu très critique de l'ouvrage de celui-ci, La stylistique ornementale dans la sculpture romane, qui paraît en 1931), ou des approches plus traditionnelles autour de Marcel Aubert?

R. R. Rappelons d'abord qu'un A. Kingsley Porter a ouvert la voie (dans tous les sens du mot) de l'étude de l'art roman aux Anglo-Saxons. Mais je dirais qu'il n'avait pas de 
2. Page du compte-rendu sur Baltrusaïtis par M. Shapiro, dans Romanesque Art, p. 276.

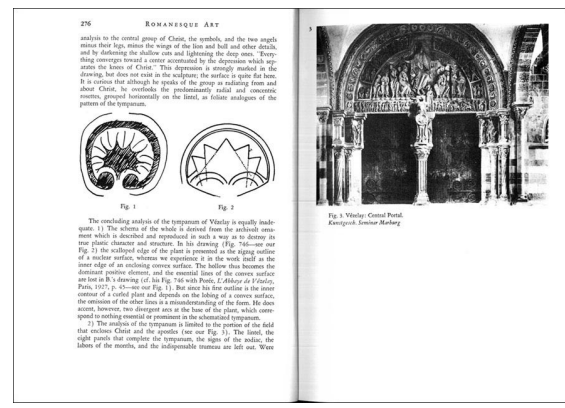

méthode. Son but était de dresser un corpus des œuvres disposées le long des "pilgrimage roads".

Meyer Schapiro entreprend cette même étude avec un objectif tout autre : il s'intéresse à la fois au contenu des œuvres sculptées et donc à leur signification iconographique - et à la forme, sachant que celle-ci est tributaire d'un matériau et d'une pensée créatrice largement déterminés par la tradition culturelle, le contexte socio-économique, etc.

Ce qui fait le lien entre ces deux exigences, ou plutôt ce qui les fonde au plan méthodologique, c'est la description. Avant Schapiro, on n'avait jamais lu une analyse des sculptures de Moissac ou de Souillac aussi précise. Elle prend en compte aussi bien le format des blocs de pierre que leurs jointoiements, la nature et les propriétés du relief, I'adaptation ou le chevauchement des scènes par rapport aux supports. Lorsque nous lisons L'art des sculpteurs romans de Focillon ${ }^{16}$, nous comprenons tout ce qui chez son collègue français a pu irriter Schapiro : le lyrisme inspiré de Focillon est à l'opposé de la clarté et de la précision, du souci didactique de Schapiro.

Mais celui-ci supportait encore moins Baltrusaïtis. Dans les Kritische Berichte, la revue des «structuralistes » viennois, une revue d'histoire de l'art des années 1930 consacrée à la méthode et à des comptes rendus critiques très élaborés, Schapiro rédige un compte rendu féroce de La stylistique ornementale dans la sculpture romane (1931) et pour ma part, je considère que la majorité de ses critiques était parfaitement fondée : Baltrusaïtis est une figure tout à fait surévaluée de I'histoire de l'art. Son formalisme apparaissait aux yeux de Schapiro comme une falsification : à la place d'une description, Baltrusaïtis livrait à ses lecteurs des schémas géométriques dans lesquels il redessinait les scènes romanes en les trahissant la plupart du temps. Une sorte de lit de Procuste... Mais Schapiro lui reprochait aussi des contresens iconographiques et un intérêt insuffisant pour cette matière. Il avait une grande admiration pour les livres d'Émile Mâle, même s'il en critiquait fréquemment tel point de doctrine ou d'interprétation.

C'est donc bien sur deux relations à l'acte descriptif en histoire de l'art, diamétralement opposées, que se fondent leurs positions respectives. Pour Baltrusaïtis, les œuvres doivent se plier au système élaboré par l'historien; pour Schapiro, I'historien, même marxiste, doit considérer l'œuvre comme préalable à toute investigation et dépassant toujours toute interprétation, quelle qu'elle soit. Pour le premier, il aurait existé une sorte $d^{\prime}$ " ordre roman » comme il y a un ordre dorique ou corinthien... Cette idée était totalement insupportable, et pour cause, à Schapiro. Quant à des personnalités un peu datées déjà dans les années 1950, comme celle d'un Marcel Aubert, elles ne l'intéressaient guère.

Il faut cependant rappeler que lorsque Schapiro s'est attelé à son sujet de thèse (Moissac) et est venu travailler en France, en 1926-27, la sculpture du Languedoc faisait l'objet de vives controverses dans la communauté scientifique française, en particulier au sujet de sa place dans la chronologie de la sculpture romane, comme le prouvent les travaux d'Émile Mâle et de Paul Deschamps. En Allemagne, Wilhelm Vöge avait déjà soulevé cette question en 1894 dans un livre que Schapiro connaissait bien et dont il semble avoir médité la méthode (Die Anfänge des monumentalen Stiles im Mittelalter).

E. C. Le rapport de Meyer Schapiro avec Arthur Kingsley Porter a été important pour les perspectives nouvelles ouvertes par des livres tels que 

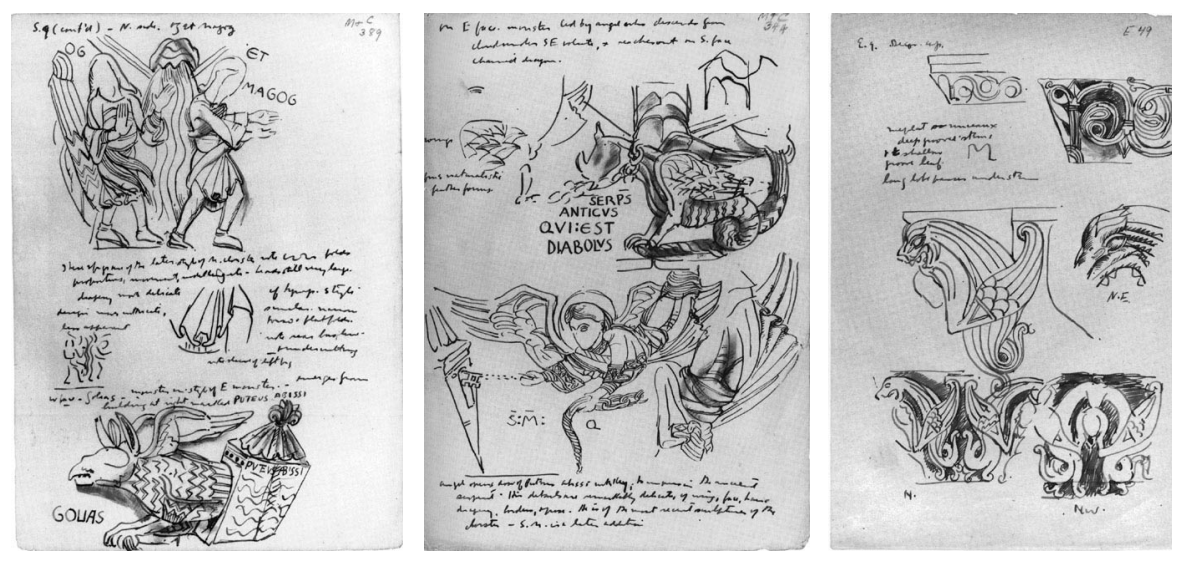

Romanesque Sculpture of the Pilgrimage Roads (1923) qui brisaient les frontières nationales et élargissaient le corpus. Les études de Charles Rufus Morey sur l'art roman et sa façon de mettre en rapport enluminure et sculpture ont également joué un rôle. Des textes d'Émile Mâle comme L'art religieux du XII siècle en France ont été très importants, encore une fois, pour les rapports établis entre enluminure et sculpture, rapports limités toutefois, comme Schapiro le fait remarquer, au niveau iconographique (et sur certains points de l'analyse iconographique de Mâle, Schapiro exprime même de fortes réserves, comme dans "Two Romanesque Drawings in Auxerre and Some Iconographic Problems »).

Schapiro était extrêmement intéressé par une lecture de l'œuvre qui mette en évidence les principes structuraux de celle-ci. Cela est très clair dans l'essai sur Souillac (1939), par exemple, mais il refusait un formalisme excessivement abstrait et surtout l'énonciation de lois trop générales qui ne tiennent pas compte du contenu et de la signification de l'œuvre et, par là, de l'histoire. D'où le compte rendu négatif sur l'ouvrage de Baltrusaïtis et la distance par rapport à Focillon (qui pour sa part ne comprenait pas grand chose au travail de Schapiro).

Pour ce qui est des "approches plus traditionnelles", il me semble que Schapiro les a utilisées largement et sans préjugés quand elles apportaient des éléments utiles à la compréhension historique de l'œuvre. Je pense de plus que l'ouvrage majeur génial de Wilhelm Vöge, Die Anfänge des monumentalen Stiles im Mittelalter (1894), a dû beaucoup retenir son attention. Â part ça, il serait long d'énumérer ce qui a intéressé Schapiro dans les méthodes de lecture de la Kunstwissenschaft allemande (qu'il connaissait très bien), de Riegl à Dagobert Frey et à la "Neue Wiener Schule", même s'il en percevait les aspects souvent contradictoires et les limites (voir son article de 1936 publié dans The Art Bulletin ${ }^{17}$ ).

R. M. Ses travaux des années 1930 se présentent à la fois comme traditionnels et innovants. D'une part, Schapiro maîtrisait tous les savoirs " scientifiques », que ce soit la linguistique, I'histoire, la liturgie médiévale, et il les a appliqués à son analyse approfondie de la sculpture de Moissac et du Sud-Ouest. Dans la mesure où cette formation était scientifique, voire philologique, son approche s'apparentait à celle de Porter et même d'une certaine manière à celles de Mâle et Aubert. Sa méthode divergeait pourtant de par une approche résolument pluridisciplinaire, nourrie de la Kunstwissenschaft allemande et des recherches faites surtout dans les sciences sociales.

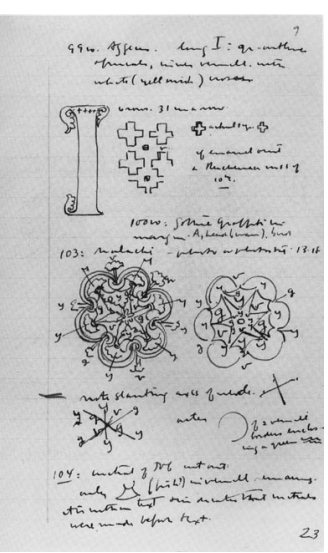

3-6. Meyer Schapiro, dessins de chapiteaux du cloître de Moissac. 
Focillon critiquait Schapiro sur ce point précis, lui rapprochant sa méthode « sociologique » qu'il confondait avec l'œuvre, en France, d'Hippolyte Taine - une remarque révélatrice de son erreur de lecture.

L'intégration de la psychanalyse, du matérialisme historique et des théories linguistiques de la communication appliquées à une approche historique qui réclamait en même temps une sensibilité moderne était fondamentalement novatrice et a valu à Meyer Schapiro une renommée certaine en très peu de temps. Dès la parution de ses études sur Moissac, Souillac, Silos et Saint-Gilles-du-Gard, c'est-à-dire avant la fin des années 1930, le jeune chercheur se rangeait parmi les médiévistes américains de tout premier ordre. Sa renommée n'a pas échappé à Focillon lors de leur unique rencontre, le gênant à un point tel que peu de temps après ce dernier n'a pas hésité à qualifier "d'usurpée " la réputation de ce "raté malheureux ${ }^{18}$.

Tous ces apports pluridisciplinaires, sans compter son intérêt pour l'art contemporain et son engagement politique, le distinguaient aussi radicalement de ses pairs médiévistes en Amérique, ce qui n'avait pour effet que d'accroître sa réputation. En revanche, en France, I'œuvre de Schapiro est presque passée sous silence dès les années 1930. Sans doute sa critique du livre de Baltrusaïtis y est-elle pour quelque chose, mais cela n'explique pas tout. Le refus de prendre en compte et de déterminer épistémologiquement les résultats de ses recherches et sa méthode révèle plutôt la division profonde, philosophique, entre les traditions anglo-saxonne et française.

Perspective. Quels ont été les principaux apports de Meyer Schapiro à l'étude de la sculpture romane? Quelles sont les pistes de recherche, les réflexions intellectuelles qui seraient encore à approfondir?

R. R. Meyer Schapiro est un historien de l'art profondément original. Il n'a donc jamais rien fait comme tout le monde. Sa pensée opérait une sorte de va-et-vient constant entre les périodes historiques parce que son intérêt le portait vers des problèmes qui dépassaient le cadre de l'Histoire. L'art contemporain, celui qui se pratiquait après la Seconde Guerre mondiale sous le regard acerbe de deux autres intellectuels marxistes new-yorkais comme Clement Greenberg ou Harold Rosenberg, a toujours intéressé Schapiro, et lorsqu'il écrit sur la sculpture romane, il cherche aussi à comprendre de quelle nature est ce "primitivisme » à l'œuvre à Silos ou à Moissac, et s'il peut contribuer à nous faire mieux comprendre notre propre temps.

On pourrait opposer deux figures ô combien antinomiques, mais que l'article que Schapiro a consacré dans The Art Bulletin à la "Neue Wiener Schule" m'autorise à comparer : celle de Hans Sedlmayr et celle de Schapiro. Pour le premier, qui est devenu dans ces mêmes années 1930 un nazi d'envergure, l'art roman est un art dégénéré : il annonce la dépravation dans laquelle serait tombé l'art moderne, notamment celui d'un Picasso. Ce serait le règne de l'irrationnel, de la fantaisie débridée. Pour Schapiro, l'art roman représente une phase archaïque de l'évolution des formes, phase que nous comprenons d'autant mieux aujourd'hui que notre intérêt nous pousse aussi vers le dessin d'enfant, etc. Il s'appuyait notamment sur les idées qu'Emmanuel Löwy avait développées à propos de l'art archaïque grec.

La question qui demeure ouverte, Schapiro le reconnaît dans l'un de ses textes, c'est celle d'une théorie du style. À travers l'étude du style, qui n'est possible qu'à l'aide d'une description exigeante, nous pouvons accéder au noyau du sens des œuvres. Mais à condition de considérer que le style ne se réduit pas à la forme. 
R. M. Un bilan des apports de sa recherche n'est pas facile à dresser, tant ils sont à la fois précis (par exemple la datation d'une œuvre ou l'éclairage d'une iconographie) et généraux (ainsi, entre autres, Meyer Schapiro proposant d'expliquer comment les forces artistiques opèrent dans la société). Ses écrits témoignent également d'une grande subtilité qu'il faut réussir à percevoir. Si l'on s'en tient à une lecture hâtive, par exemple, de son essai sur le retable de Mérode ${ }^{19}$, il ne ressemblerait plus qu'à un exercice d'application de l'iconographie panofskienne, alors qu'il en constitue une critique de base... De plus, certaines de ses convictions, entre autres celles qui prônent I'individualité créative avant tout et une sensibilité formaliste à la Greenberg, ne trouveront que des échos assourdis pour nous, ces positions étant trop proches du « modernisme » pour nos scepticismes « post-modernes » contemporains. Or ce même type de réflexions le conduisait à une lecture de l'œuvre d'art qui prenait en compte à la fois la forme et le fond, comme préalable indispensable à toute explication de I'œuvre, une interrogation qui reste d'actualité.

Cette conviction le conduisait à s'interroger sur les capacités de la forme à être porteuse de significations, celles-ci s'articulant bien évidemment à l'iconographie mais s'étendant au-delà du sujet iconographique à proprement parler. Le principe de "coordinated discoordination" qu'il a développé dans son essai sur Souillac ${ }^{20}$ en est une démonstration magistrale : il voyait dans certaines qualités formelles qui semblaient ne pas être " coordonnées » (c'est-à-dire organisées selon une logique apparente) et certains éléments iconographiques qui, eux non plus, ne semblaient pas être organisés selon une correspondance habituelle, une manière significative. Les « discordances " sont donc pleines de sens. Vu sous un autre angle, il a traité le relief de Souillac, une œuvre figurative, rappelons-le, comme une œuvre également chargée de qualités abstraites, et par ce procédé il a pu par la suite allier les forces significatives que l'on pense souvent propres à ces deux types de production artistique différents. Cette notion de "discordance " est au fond dialectique, et sa réflexion sur la dialectique l'a sensibilisé à des notions analytiques comme "process", "indeterminacy" et "fragmentation", notions qui sont au cœur de débats théoriques de plusieurs disciplines aujourd'hui.

Et alors que de tels aspects intéressent toujours des chercheurs, d'autres concepts de la pensée marxiste repris par Schapiro dans ses écrits n'ont pas aujourd'hui le même retentissement qu'à son époque. Cependant, il est bon de rappeler que s'il croyait en certains points seulement du matérialisme dialectique, il n'était pas un marxiste doctrinaire. II ne voyait en aucun cas le marxisme (un terme jamais prononcé dans ses travaux sur l'art médiéval) comme un système à appliquer à tout prix. Cette prise de conscience politique lui a néanmoins permis de démontrer l'importance d'une enquête sur les liens réciproques entre les conditions sociétales et les forces créatrices. Dans son essai sur Silos ${ }^{21}$ (peut-être le plus marxiste de ses écrits sur le Moyen Âge), il a même précisé que "des recherches futures changeront peut-être certaines de ses conclusions, mais auront à suivre la même méthode que celle développée ici, selon laquelle on prend en compte les rapports critiques entre les formes et les significations des images avec les conditions historiques de la région à la même époque » ${ }^{22}$. Tout un programme à suivre.

E. C. Je crois qu'un apport très important de Meyer Schapiro à l'étude de la sculpture romane a été de lire les monuments et les variations stylistiques à la lumière de la situation historique et culturelle et de ses conflits (voir le texte sur la Ruthwell Cross ${ }^{23}$ 
7. Couverture de La sculpture de Moissac, Paris, 1987.

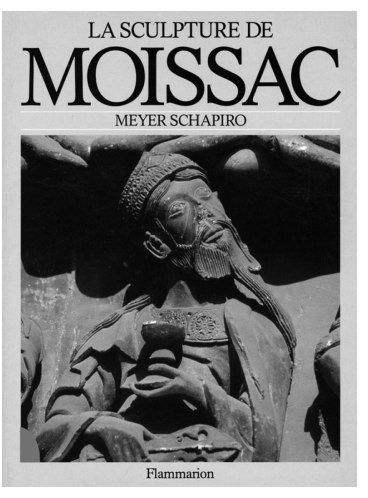

ou celui sur Silos) où les styles mozarabe et roman sont vus dans un contexte très riche, tout comme le cas de Souillac avec les conflits entre anciennes revendications ecclésiastiques et nouveaux rapports sociaux). Cette lecture est autrement complexe que les simplifications habituelles des adeptes de la théorie du reflet. Loin de chercher le reflet, Schapiro cherche à lire les différentes facettes du prisme. Sa méthode, résolument empirique, est une exemplification du fait que «le bon Dieu est dans le détail ». L'attention au détail va de la lecture des inscriptions à celle des simples (et mystérieuses) lettres isolées, des considérations sur l'opposition entre marges et centre (entre les images à l'intérieur du tympan et celles sur les consoles), entre abstraction et réalisme, entre symétrie et contrapposto, entre archaïsme et naturalisme. À chaque fois, Schapiro s'interroge sur les raisons qui ont fait choisir une solution plutôt qu'une autre et ne cherche pas à imaginer des lois universelles. Très importante aussi est l'attention constante portée à l'artiste, à ses méthodes de dessin, à ses conditions de travail (chapiteaux sculptés à pied d'œuvre ou sur place), à sa fantaisie. II s'intéresse également au rapport, variable, des personnages avec le cadre, ce qui contredit toute généralisation normative. II faudrait ajouter l'ampleur de l'enquête iconographique dont il est capable, qui peut l'amener à des constatations très nouvelles, comme dans le cas exemplaire de deux dessins d'Auxerre.

Par conséquent, ce qui me semble particulièrement vivant, et digne de suite, chez Schapiro, c'est sa lecture des innombrables facettes du prisme, une lecture à 360 degrés.

Perspective. Les écrits de Meyer Schapiro ont presque tous été réédités aux États-Unis, notamment ceux sur la sculpture romane (Romanesque Art. Selected Papers, 1977, rééd. 1993). Une courte sélection d'articles, portant principalement sur la notion de style et les approches de l'art liées aux sciences humaines, a été publiée en français en 1982, sans inclure aucun texte sur l'art roman. Encore récemment, la journée consacrée à Meyer Schapiro à l'Auditorium du Louvre à l'occasion de l'exposition La France romane (un titre, il est vrai, que n'aurait sans doute guère aimé Meyer Schapiro) était surtout orientée vers sa pensée sur l'art contemporain. Comment expliquer l'absence de réception en France des études de Meyer Schapiro sur la sculpture romane, dans les années 1930, mais aussi à l'époque actuelle? Parce qu'il était juif? Par un discours trop proche, aux oreilles françaises, de celui de Focillon, pour la loi du cadre ou la notion de style comme expression d'une civilisation? Par une pensée trop orientée, comme le montre parfois son article "On the Aesthetic Attitude in Romanesque Art ", vers une approche purement moderniste, américaine, de l'histoire de l'art, non éloignée du discours du critique Clement Greenberg ? E. C. Tout d'abord il me semble qu'un texte au moins de Schapiro sur la sculpture médiévale, celui sur Moissac, a été traduit en français (La Sculpture de Moissac, 1987). Quant à la proximité de Schapiro par rapport à Focillon, elle n'est qu'apparente : en réalité la méthode de Schapiro est fort différente de celle de Focillon et la lecture "formelle» (conduite d'ailleurs d'une autre façon que celle de Focillon) ne constitue qu'un élément dans une enquête bien plus vaste qui engage iconographie, histoire sociale et culturelle, technique etc. Aussi la notion de style comme expression d'une civilisation ne me semble-t-elle pas pertinente pour l'approche "prismatique » de Schapiro. Je ne pense pas d'autre part que le fait d'être juif ait pu jouer un rôle dans cet 
oubli, étant donné que pour d'autres intellectuels juifs cela ne s'est pas produit. Je verrais plutôt deux ou trois causes possibles : I'intérêt pour le Schapiro sémiologue - ou mieux, sémiologue d'une façon explicite -, I'intérêt pour le Schapiro « contemporanéiste » ont primé sur le Schapiro médiéviste sans qu'on comprenne le rapport qui existe entre les deux. II faudrait aussi réfléchir sur l'équivoque d'un Schapiro « déconstructionniste » et sur l'interprétation qui a été donnée au terme de "discoordination" employé par lui dans l'essai sur Souillac.

J'ajoute que je vois mal un rapport entre le discours de Greenberg et l'essai « On the Aesthetic Attitude in Romanesque Art » qui cherche à mettre en lumière les formes de la réception et les hiérarchies de l'art médiéval aux yeux des contemporains.

Pour conclure, je dirai que c'est la richesse et la complexité même de la méthode ( " prismatique ») de Schapiro qui peuvent avoir éloigné la réception française du Schapiro médiéviste et "spécialiste » de la sculpture médiévale française, spécialisation qu'on a pu considérer, à tort ou à raison, comme une spécialité " hexagonale ». D'ailleurs, pour confirmer mon impression, je constate que Vöge, un sommet dans I'historiographie de la sculpture médiévale française, n'est pas très connu en France.

R. M. Comme vous le suggérez, l'absence de traductions joue un rôle important et il est regrettable que la grande majorité de ses écrits ne soient pas à la portée des lecteurs francophones.

L'absence de réception en France s'explique sans doute en partie par le compte rendu du livre de Baltrusaitis. Sa critique, perçue en France comme une attaque contre Focillon, a sûrement retenti auprès des médiévistes français, très fidèles à leur maître. Focillon I'a en tout cas perçue comme telle, décrivant Schapiro par la suite comme " infecté de la méthode germanique ». Dans cette phrase, " germanique », comme l'a précisé W. Cahn, se rapporte à une pensée trop philosophique, voire obscurantiste, au goût de Focillon, mais le mot était bien sûr chargé d'autres significations dans les années $1930{ }^{24}$. Une méprise, donc, de l'apport de la philosophie à l'art médiéval, dont l'étude en France était fondamentalement enracinée dans l'archéologie monumentale. En dénonçant dans son compte rendu un formalisme trop mécanique, aléatoire, et anhistorique à ses yeux, les objections de Schapiro ont peut-être eu pour effet secondaire une distorsion de son propre projet auprès des chercheurs français, qui ne verront en lui qu'un adversaire. Schapiro prônait pourtant l'importance d'une analyse formelle.

Il est aussi vrai qu'en France on était en présence de deux camps : d'un côté les adeptes de l'iconographie et de l'autre l'école «focillonienne ». Même si l'iconographie et le formalisme existaient aux États-Unis et en Angleterre comme en France, les travaux de Schapiro ont déblayé une autre voie. Il a su tirer le meilleur parti de chaque méthode et par conséquent les transformer l'une et l'autre. II n'est peut-être pas exagéré de dire que l'iconographie exercée par maints médiévistes anglo-saxons doit plus à Schapiro qu'à Panofsky (même s'ils ne suivent pas de très près Schapiro dans son vocabulaire marxiste, psychique...) Cela ne me semble pas le cas en France, d'où peut-être le clivage méthodologique que nous vivons actuellement.

En dernier lieu, l'absence de réception en France est due peut-être en partie à une réticence plus générale pour l'historiographie médiévale, et cela en dépit de nombreuses études signées par mes deux collègues dans ce débat, Roland Recht et Enrico Castelnuovo. Dans d'autres pays, I'historiographie a pris une ampleur considérable ces dernières décen-

8. Meyer Shapiro, Abstraction, formes sur une table, 1940.

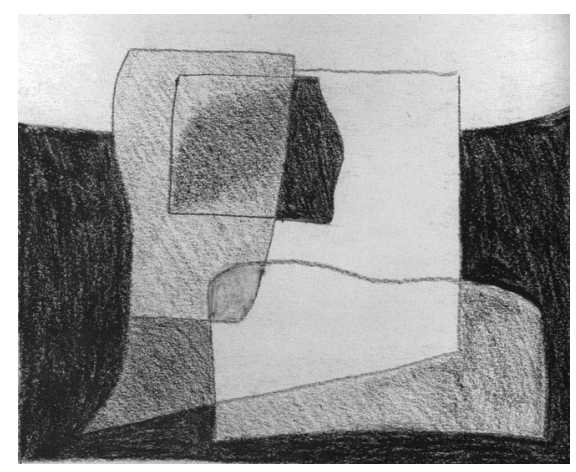




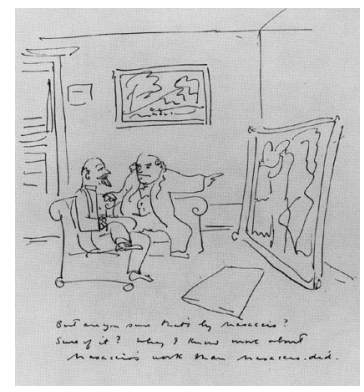

9. de Meyer Shapiro, Monsieur Berenson et le collectionneur, 1940. nies (démesurée, selon certains), alors qu'elle demeure peu suivie par les historiens $d^{\prime}$ art en France, pour en rester encore dans le domaine de la philosophie ou de I'histoire de l'esthétique. L'enjeu de l'historiographie, bien sûr, n'est pas simplement une meilleure compréhension de tel ou tel historien ou théorie, mais une prise de conscience des bases épistémologiques de notre discipline, à partir desquelles on puisse repenser les fondements de notre praxis et renouveler l'histoire de l'art d'aujourd'hui.

R. R. Quelle vaste question que celle de la non-réception en France des œuvres de Schapiro! Plus exactement, de sa réception sélective, faussée, pour ne pas dire falsifiée. C'est un chapitre considérable d'un livre encore plus considérable qu'il conviendrait de consacrer à toutes les non-réceptions qui sont une singularité française... De petits groupes de pression, des éditeurs puissants sont à l'origine de ces phénomènes, indépendamment de la valeur scientifique des travaux traduits et mis à la portée du public francophone. De Haskell et de Baxandall, qui sont de grands historiens de l'art, on ne s'est pas contenté de traduire que les bons livres... Les écrits de Julius von Schlosser les plus importants ne sont pas sa Kunst- und Wunderkammer, bien dépassée depuis longtemps, mais ceux qui portent sur la mémoire des images, assez proches de Warburg par certains côtés : mais pour le savoir, il faut lire ses articles très longs parus dans des revues peu accessibles en France. Et les éditeurs n'ont pas beaucoup d'imagination... L'instrumentalisation actuelle de Warburg en France est une autre affaire qu'il faudrait traiter un jour dans le détail (où l'on sait que ne se cache pas seulement le Bon Dieu !)

Dans les années 1970, certains milieux intellectuels en France, après avoir tué le père, avaient besoin de lui substituer d'autres figures tutélaires, non-françaises souvent, pour légitimer leurs propres positions théoriques : une partie de l'œuvre de Panofsky a été ainsi instrumentalisée par la sociologie bourdieusienne, mais aussi par Foucault dans un article fameux du Nouvel Observateur (25 oct. 1967). Si j'évoque Panofsky, c'est que Bourdieu voyait en lui un modèle structuraliste ; les introducteurs de Schapiro en France joueront sur le même registre. Son article consacré à « Quelques problèmes de sémiotique de l'art visuel [...] », paru en 1969 dans la revue Semiotica qui venait d'être fondée comme organe de l'Association internationale de sémiotique présidée par Émile Benveniste, est traduit dans Critique l'été 1973. Cet article n'est de loin pas le meilleur de Schapiro. Mais il traitait avec une largeur de vue habituelle chez son auteur de problèmes rattachés de ce côté-ci de l'Atlantique au matérialisme dialectique, qui travaillaient alors un certain nombre d'artistes et de théoriciens ou de critiques d'art, mais qui semblaient aussi pouvoir éclairer certaines pratiques picturales d'Américains comme Robert Ryman ou même Pollock.

Schapiro a été « utilisé » dans le cadre d'une connivence qui s'était établie dans les années 1970 entre la revue new-yorkaise October et la revue Macula, qui avait l'ambition de devenir une sorte d'October français. Le $n^{\circ} 3-4$ (1978) contient un dossier intitulé "Heidegger et les souliers de Van Gogh » où paraît le court essai de Schapiro de 1968.

Cette vision d'un Schapiro sémiologue est aussi fausse que celle d'un Schapiro marxiste pur et dur ou encore d'un Schapiro freudien orthodoxe. Il a abordé toutes ces questions avec une infinie prudence et avec une grande méfiance à l'égard de tout " système » d'interprétation. Mais ces intérêts allant vers des champs de réflexion écartés de l'histoire de l'art orthodoxe française, ce sont en même temps trois raisons 
pour lesquelles Schapiro n'a pas reçu en France l'accueil qu'il méritait. C'est donc un petit groupe obnubilé par le modernisme greenbergien qui a tiré Schapiro à lui : ces gens-là n'avaient rien à faire de Moissac et de Silos, du moins le croyaient-ils, ces malheureux! Ils ignoraient que Schapiro lui-même avait écrit sur l'art roman en pensant à l'art moderne.

Willibald Sauerländer a très judicieusement appelé Schapiro un « outsider » : il I'était dans la discipline, dans son propre pays et a fortiori vu depuis Paris ! Un marxiste non-doctrinaire et anti-stalinien dans les années 1940-50, autant à l'aise dans les subtilités iconographiques des enluminures byzantines que dans les entrelacs de I'herméneutique freudienne, c'est quand même pas si mal, non?

Perspective. Quel a été votre occasion de rencontre avec les écrits de (ou l'homme) Meyer Schapiro. Comment avez-vous utilisé, avez-vous pu vous approprier ses " méthodes " pour vos propres travaux?

R. M. J'ai découvert ses écrits jeune, puisqu'un de ses essais nous était donné à la fac, dans un cours de licence très général sur l'art du Moyen Âge. Mon expérience n'a sûrement pas été exceptionnelle, mais typique de l'enseignement du Moyen Âge dans les facultés américaines; c'est pour vous dire à quel point Schapiro est au cœur de I'histoire de l'art outre-Atlantique. Je les ai redécouverts quelques années plus tard quand j'ai recommencé mes études, en maîtrise, car ses écrits faisaient également partie du cursus, et je les ai étudiés encore plus tard pendant que je rédigeais ma thèse. Aujourd'hui je les donne à mon tour à mes étudiants tous les ans, et non seulement dans les cours sur le Moyen Âge (de tous niveaux, de la licence jusqu'au doctorat) mais aussi dans les cours sur la méthodologie et les théories de l'art. À chaque occasion de relecture, j'ai l'impression de redécouvrir à nouveau Schapiro, tellement ses écrits sont riches en réflexions et pistes prometteuses de recherches futures. Or, imiter Schapiro est inutile : ce ne sont pas simplement ses connaissances, son raisonnement et sa perspicacité méthodologique qui le mettent à part, mais avant tout sa créativité foisonnante. La sagesse avec laquelle il a su allier subtilement une créativité intellectuelle à un empirisme à la fois rigoureux et philosophique pour faire de l'histoire de l'art une expression de la critique est en fin de compte inimitable. C'est cependant un modèle auquel notre discipline devrait peut-être plus aspirer.

R. R. Parce que vous me posez une question très personnelle, permettez-moi un écart autobiographique. Quantitativement, j'ai consacré environ vingt ans de mon activité d'historien de l'art au Moyen Âge, et vingt autres années à fouler d'autres continents à partir desquels je n'ai cessé de lorgner régulièrement vers le Moyen Âge. Mais ces années ne se sont pas succédé mais entrecroisées. Dans les années 1960, consécutivement à la lecture de Foucault et de Lévi-Strauss, je cherchais dans la sémiologie et dans l'anthropologie structurale s'il n'y avait pas là moyen de bricoler un modèle pour I'histoire de l'art. Mais ça ne collait pas. La scénographie d'un tableau de Schefer (dans la mouvance de Tel Quel) était assez éblouissante, mais me paraît avec le recul un peu laborieuse. Et malgré les qualités des travaux d'un Louis Marin ou d'un Hubert Damisch, je n'arrivais pas à me convaincre qu'ils tenaient le bon bout. Les tentatives venues du côté de la psychologie ou de la psychanalyse freudienne pour fonder un discours sur l'art qui fût pertinent réintroduisaient le sujet, la figure de l'artiste, là où I'on croyait que Foucault l'avait évacuée. Et ma première lecture de Meyer Schapiro, 
fut en 1968 dans les Renaissance Essays réunis par Paul Oskar Kristeller, son article sur Léonard et Freud ${ }^{25}$ : il percevait avec une acuité remarquable l'écart entre la psychanalyse comme science et l'échec du Souvenir d'enfance, tout en réaffirmant avec fermeté la nécessité de réintroduire l'Histoire. À la même époque, je lisais beaucoup Panofsky et, par la force des choses, aussi ses critiques les plus éminents, à savoir Otto Pächt et Meyer Schapiro - I'article de ce dernier sur le retable de Mérode (Muscipula diaboli) dans un vieux de The Art Bulletin (1945) que m'avait signalé Grodecki, je crois. J'ai déjà évoqué le dossier de Macula et un article qui m’avait beaucoup impressionné, c'était Les pommes de Cézanne paru dans le premier numéro de la Revue de l'Art lancée par André Chastel en 1968 (ce qui éclaire une réception française de Meyer Schapiro très précoce). Je n'ai pu avoir une vue d'ensemble de la valeur des travaux de Meyer Schapiro qu'en acquérant en 1979 ses trois volumes de Selected Papers. Ce n'est donc pas en tant que médiéviste que j'ai d'abord été mis sur la voie de ses travaux. Mes intérêts dans ce domaine se situaient plutôt dans les années 1965-70 du côté de l'architecture, que Meyer Schapiro n'a jamais abordée de front (il l'avait nécessairement étudiée en travaillant sur la sculpture romane) et là, les Américains avec lesquels je menais des discussions s'appelaient par exemple Robert Branner, qui ébranlait fortement l'hégémonie des historiens de l'art germanophones, formalistes, comme Jantzen, ou « iconologues» comme Sedlmayr et Von Simson, dans l'étude de I'architecture gothique, sans échapper à un certain positivisme. Branner et Meyer Schapiro qui étaient collègues à Columbia s'ignoraient superbement. Ils ne faisaient pas le même métier ! Je dirai donc qu'à ce moment-là les travaux de Meyer Schapiro, comme ceux d'un Cesare Brandi ou d'un Aby Warburg pour m'en tenir à des auteurs peu orthodoxes au sein de la discipline en France, m'ont appris à réfléchir autrement sur l'histoire de l'art en général et accessoirement sur des objets qui étaient les miens, produisant parfois des effets concrets après bien des années. Dans ma thèse d'État sur la sculpture du gothique tardif, en 1978, je ne pense pas avoir cité Meyer Schapiro une seule fois ; mais ma préoccupation pour lier sans cesse l'œuvre à ses conditions de production, c'était quelque chose qui était « dans l'air » à cette époque-là, et n'était plus le propre de Meyer Schapiro qui avait été un précurseur trop en avance, dirais-je. Mais qui saura dire l'impact qu'ont nos lectures, à un moment déterminé de notre travail, dans tel ou tel champ, sur notre réflexion prise dans la longue durée ? Leur effet $s^{\prime}$ entrecroise avec d'autres lectures et le tout finit par constituer notre petite singularité propre.

E. C. Je ne sais pas au juste à quel moment j'ai rencontré Meyer Schapiro ou, mieux, ses écrits. Je me rappelle bien les discussions soulevées en Italie par son article très critique «I valori di Berenson» (1961) qui était une prise de position méthodologique plus que moraliste. Je me rappelle aussi que j'avais photocopié son article «Style » ${ }^{26}$ pour m'en servir dans les discussions du séminaire quand à l'époque j'étais assistant à Turin et encore plus pendant mon enseignement à Lausanne à partir de 1964. Mais c'est surtout à travers deux essais et deux comptes rendus que je suis entré en contact avec la pensée et la méthode de Meyer Schapiro. Les essais étaient "On the Aesthetic Attitude in Romanesque Art » et " From Mozarabic to Romanesque in Silos », les comptes rendus, très critiques, étaient ceux consacrés au livre de Baltrusaïtis (paru dans Kritische Berichte de 1932-33) et celui sur la " Nouvelle école de Vienne » publié dans The Art Bulletin en 1936. " On the Aesthetic Attitude » m'avait ouvert des portes à la lecture de l'art médiéval, m'avait fait comprendre que la hiérarchie des valeurs artistiques pour 
I'homme du Moyen Âge était fort différente de la nôtre. Ce texte permettait d'approcher la sensibilité visuelle d'un spectateur du XII ${ }^{\mathrm{e}}$ siècle qui pouvait être bien plus impressionné par les couleurs, la matière, la « texture », la richesse et les dessins d'une étoffe qui enveloppait le corps d'un saint, vue le jour de la translation des reliques, que par une fresque qui décorait les parois d'une église. Je commençai à regarder d'une façon différente les tissus qui se trouvaient dans les trésors des églises en essayant de comprendre quelles sensations ils pouvaient susciter. Cet essai était une introduction aux problèmes de la réception et répondait à mes préoccupations sur le danger $d^{\prime}$ utiliser uniquement nos critères et nos valeurs dans la lecture d'une œuvre médiévale en ignorant la façon de voir et de juger des contemporains, une habitude qui pouvait nous cacher bien des choses. Dans l'essai sur Silos j'étais fort intéressé par la façon, très subtile et pas du tout schématique, de mettre en rapport des innovations stylistiques avec une situation culturelle et sociale particulière. Dans les années 1970 j'ai de plus en plus fréquenté les écrits de Schapiro et si je regarde les notes de bas de page de mes articles sur l'histoire sociale de l'art parus en 1976-77, je trouve bien des références à ses textes qui à l'époque n'étaient pas encore réunis dans les Selected papers. Le volume des écrits de Meyer Schapiro consacré à l'art roman, Romanesque Art, publié aux États-Unis en 1977, a été traduit en italien (Arte romanica) par Adriano Sofri, le capitaine Dreyfus de notre temps, et publié par Einaudi comme numéro 641 de sa collection «Saggi » en 1982. Assez tôt (je dirais même très tôt) donc, d'autant plus que les écrits réunis dans ce volume ne concernaient pas I'art roman en Italie. Je collaborais à l'époque avec la maison Einaudi mais je ne me rappelle pas très bien qui l'avait signalé à l'éditeur, peut-être Cesare Segre, philologue roman et sémiologue qui préfacera le volume des écrits de Schapiro sur l'art moderne publié par Einaudi peu d'années après ${ }^{27}$, ou le contemporanéiste Paolo Fossati, ou Carlo Ginzburg, ou moi-même, car j'avais depuis longtemps une grande admiration pour Schapiro, ou encore tous les quatre. Une nouvelle édition parut en 1988, toujours chez Einaudi, mais cette fois dans la Biblioteca di Storia dell'Arte ( Nuova Serie», $n^{\circ} 3$ ). En tout, en additionnant les deux éditions, sept mille exemplaires, ce qui est un beau chiffre. Le volume est depuis longtemps épuisé et j'espère qu'il sera un jour ou l'autre réimprimé.

Perspective. Les études sur l'art roman semblent avoir subi un profond renouvellement depuis une vingtaine d'années. Comment, dans chacun de vos pays respectifs, les nouvelles enquêtes prennent-elles en compte les méthodes, les questions de Schapiro, tant pour la sculpture que pour l'iconographie ou l'étude de la civilisation médiévale?

E. C. La notoriété de Meyer Schapiro en Italie n'a pas connu d'éclipses, mais, comme ailleurs, c'est le Schapiro « sémiologue » qui a surtout retenu I'attention du public et rarement on a fait la liaison entre le Schapiro médiéviste et le Schapiro contemporanéiste. Toutefois, récemment, la longue et riche introduction de Giovanna Perini à un recueil d'essais de Schapiro qu'elle a traduits arrive savamment et brillamment à joindre les deux voies ${ }^{28}$.

Les essais du Schapiro médiéviste qui ont particulièrement retenu l'attention des lecteurs italiens ont été tout d'abord "On the Aesthetic Attitude in Romanesque Art » dont l'indiscutable fortune dans la péninsule s'explique par l'attention, bien italienne, portée depuis Benedetto Croce, Lionello Venturi ${ }^{29}$ et Roberto Longhi ${ }^{30}$ à I'historiographie de la critique (voir par exemple Rosario Assunto, La critica d'arte nel pensiero 
medievale, Milan, 1961) ainsi que «From Mozarabic to Romanesque in Silos ». Dans ce dernier cas, le fait d'avoir présenté un conflit entre deux styles en le rattachant à un conflit social et culturel (comme dans le cas du texte sur la Ruthwell Cross ou de celui sur Souillac) a particulièrement intéressé les tenants d'une histoire sociale de l'art. Il est clair d'autre part que des textes comme les critiques adressées à Baltrusaïtis et implicitement à Focillon ou à la Nouvelle école de Vienne ont retenu l'attention des "storici della critica" tout comme The Parma Ildefonsus est régulièrement cité par les historiens de l'enluminure ou de la peinture romane. L'article «A Relief in Rodez and the Beginnings of Romanesque Sculpture in Southern France », avec la distinction qu'il propose pour le $\mathrm{Xl}^{\mathrm{e}}$ siècle entre sculpteurs en marbre spécialisés dans la création d'autels et de certains éléments de la décoration intérieure des églises et sculpteurstailleurs de pierre chargés de la Bauplastik de l'édifice, est connu et parfois utilisé sans toutefois de grandes conséquences au niveau méthodologique.

À mon avis, on pourra réellement se servir des instruments créés par Schapiro quand on arrivera à comprendre l'unité et la cohérence de sa recherche sans trop faire de distinctions entre médiéviste, moderniste, contemporanéiste et sémiologue. Autrement, on continuera à puiser quelques éléments ici ou là, ce qui n'est déjà pas mal !

R. M. Est-ce que les études sur l'art roman ont subi un réel renouvellement ? Le nombre de thèses soutenues aux États-Unis sur l'art roman a diminué considérablement il y a vingt/vingt-cinq années et a connu seulement un faible rebond récemment. D'après mes collègues français, la France a connu le même sort. Ce déclin persiste en France malgré les efforts passionnés de plusieurs professeurs de haute distinction spécialisés en art roman ; il est à noter qu'aucun spécialiste n'enseigne dans une faculté de l'université de Paris depuis longtemps.

Il faut remonter dans le temps pour comprendre le déclin qui a marqué les années 1980-90. L'intérêt outre-Atlantique pour l'art roman a connu son premier grand essor dans les 1920 et 30, une période pendant laquelle les plus grands collectionneurs et musées constituaient leur collections (J.P. Morgan, G. G. Barnard, les musées de Boston, New York et Philadelphie) et certains professeurs très renommés enseignaient I'art médiéval et surtout I'art roman (Porter à Harvard, Schapiro à Columbia et Morey à Princeton). Un deuxième essor plus généralement pour l'art médiéval (mais réservant une place privilégiée à l'art roman) s'est produit dans les années 1950-70, s'appuyant une fois de plus sur des expositions importantes. Le International Center for Romanesque Art a été créé. Il va devenir quelques années plus tard l'organe que l'on connaît aujourd'hui, le International Center of Medieval Art, mais il convient de rappeler que les premiers volumes de sa revue scientifique, Gesta, ont été publiés sous son auspice et donc ont été consacrés à l'art roman. Et l'enseignement de l'art médiéval a connu un bond, à cette époque, dans les plus grandes facultés américaines, d'où la formation $d^{\prime}$ une nouvelle génération de chercheurs. Celle-ci comptait les plus grands spécialistes, dont nombreux exercent encore. Leurs travaux ne se bornaient pas à l'art des $\mathrm{XI}^{\mathrm{e}}$ et $\mathrm{XII}^{\mathrm{e}}$ siècles : I'enseignement chez Schapiro ne se limitait pas uniquement à l'art roman et c'était également le cas chez les autres plus grands professeurs médiévistes de I'après-guerre, qui avaient tous une vision de l'art médiéval très large (R. Krautheimer, E. Panofsky, H. Bober, E. Kitzinger). La formation consistait bien sûr en un approfondissement des connaissances des œuvres, mais également, voire avant tout, en une approche méthodologique. Leurs maîtres, des émigrés allemands pour la plupart, prônaient une approche plus philosophique et pluridisciplinaire que l'on mettait en œuvre en étudiant des objets et des sujets précis. 
Certes, c'était le cas chez certains chercheurs en France (pour ne citer que Louis Grodecki), mais l'art médiéval en France était resté médusé par un schisme, si j'ose dire, entre l'école «focillonienne » d'un côté et les chartistes de l'autre. Ce clivage est heureusement moins présent aujourd'hui, mais on parle toujours de ceux qui s'intéressent plus à la théorie et ceux qui sont plus portés vers l'étude " archéologique » d'une œuvre (comme si l'archéologie n'avait pas de bases théoriques !) Qu'une telle distinction puisse exister montre bien que certains ont encore du mal à intégrer l'étude approfondie d'une œuvre avec des interrogations «théoriques ».

Dans les années 1970, toute la discipline de l'histoire de l'art s'est réorientée d'une manière spectaculaire pour se mettre en phase avec le renouveau théorique de I'après-68; cependant, pour l'art médiéval aux États-Unis, cette réorientation a été moins décisive. Après tout, les travaux de Meyer Schapiro, considérés depuis longtemps comme des modèles à suivre, avaient déjà frayé leur chemin et démontré l'importance d'approches nourries aux sciences sociales. D'autres grands historiens, tels Krautheimer, travaillaient aussi sur des approches contextualistes. Néanmoins, à partir de la fin des années 1970 et durant toutes les années 1980, on a pu noter une progression sensible pour ce qui concerne certaines questions méthodologiques, celles qui portaient plus particulièrement sur la linguistique, les rapports texte/image, le mécénat, la dévotion privée et le mysticisme, et qui trouvaient plus de réponses dans le monde gothique, surtout dans I'art des XIII ${ }^{\mathrm{e}}$ et XIV ${ }^{\mathrm{e}}$ siècles. L'art gothique frappait les sensibilités éveillées à de nouvelles positions théoriques, surtout les manuscrits avec leurs figures loufoques aux comportements scatologiques et leurs textes écrits en langue vulgaire (et donc qui interpellent le lecteur d'une autre façon) ou adressés explicitement à telle ou telle femme de la cour royale. L'art roman pouvait sembler au premier abord plus sobre et moins propice à certaines de ces interrogations. Et c'est effectivement dans les années 1980 que l'on remarque une baisse significative du nombre de thèses soutenues sur l'art roman. Or, comme l'a très bien montré Michael Camille dans un bel article sur Meyer Schapiro ${ }^{31}$, les travaux de Schapiro sur l'art roman traitaient de plusieurs questions similaires (linguistique, rapport texte/image, problèmes posés par les images dans les marges...) Beaucoup de ces interrogations théoriques ont en effet leur place dans l'étude de l'art roman aujourd'hui et pourraient constituer les champs d'un renouvellement futur pour son étude ${ }^{32}$. Par ailleurs, certaines questions de I'histoire sociale aux Xl $\mathrm{e}^{\mathrm{e}}$-xII ${ }^{\mathrm{e}}$ siècles que Schapiro abordait et qui trouvaient un écho dans les discours des années 1970 jouissent d'un regain d'intérêt dans la nouvelle optique socio-historique d'aujourd'hui.

Si je n'ai pas en fin de compte répondu à la question posée, c'est peut-être parce que, ainsi formulée, elle supposait qu'il y avait une " méthode Schapiro ». Or, comme le signalent mes collègues plus haut, il n’y a jamais eu de "méthode Schapiro », si ce n'est une approche fondée sur l'étude approfondie d'une œuvre qui rende compte de ses formes, son matériau, ses créateurs, et ses significations. À l'inverse de certaines méthodes dans le domaine de la recherche iconographique, qui tentent à établir d'abord le sujet pour émettre ensuite des hypothèses sur les significations de l'œuvre, Schapiro considérait l'iconographie non pas comme la source de significations mais comme un élément, parmi d'autres, de la signification plus large de l'œuvre. Autrement dit, l'iconographie n'explique pas le sens de celle-ci; c'est un indice qui laisse entrevoir les significations possibles. Schapiro ne cherchait pas par ailleurs à remettre une œuvre dans son contexte d'origine (une opération épistémologiquement dépour- 
vue de sens), mais à faire resurgir une œuvre de son contexte afin de mettre en relief " l'espace » qu'habitait l'œuvre dans le temps et dans la société.

R. $\mathbf{R}$. J'espère que mon collègue Robert Maxwell ne m'en voudra pas si je me permets de faire une brève incursion sur le sol américain dont il a très bien analysé la situation. Pour rappeler seulement que dans les années 1970-80 a été réalisé le corpus de la sculpture romane dans les collections publiques américaines, publié peu à peu dans la revue des médiévistes Gesta, puis réuni en livre ${ }^{33}$; et souligner ainsi le rôle joué par cette revue dans la diffusion des travaux sur l'art roman. Il est indéniable qu'une telle entreprise ne pouvait que stimuler l'attrait que de nombreux jeunes universitaires américains éprouvaient pour l'art du Moyen Âge en général.

En ce qui concerne la situation française, il faudrait peut-être rappeler que les débuts de l'art roman ont constitué un enjeu idéologique considérable dans les années 1930. Pour Focillon, il s'agissait de montrer que la sculpture du début du $\mathrm{XI}^{\mathrm{e}}$ siècle exprimait, même maladroitement, la première manifestation de cette "loi du cadre » qui dominait, selon lui, la représentation figurée romane et dont allait ensuite s'affranchir I'« humanisme» gothique. Dans un livre intitulé L'humanisme roman (1942), Pierre Francastel, prenant pour modèle La société féodale de Marc Bloch (1939) et le contre-pied des thèses de Focillon, cherche à interpréter le Xl ${ }^{\mathrm{e}}$ siècle comme l'aube de la formation de l'unité française. Francastel ignorait tout des travaux de Meyer Schapiro mais avançait d'une façon très péremptoire des positions arrêtées sur l'art roman, plus préoccupé de polémiquer que d'approfondir ce champ de recherche qui ne lui était guère familier.

J'en viens maintenant à une époque plus récente. Dans les années 1950-70, l'organe de la Société française d'archéologie, le Bulletin Monumental, dirigé par Marcel Aubert puis par Francis Salet, publie de nombreuses études sur l'art roman auxquelles il faut évidemment ajouter la parution annuelle des Congrès archéologiques émanant de la même instance. Nous sommes là dans la tradition chartiste mais il est indéniable que le formalisme d'un Focillon a contaminé bon nombre d'esprits attachés d'abord aux sources textuelles (Salet se comporte parfois comme un « attributionniste » redoutable d'intransigeance !) On doit rappeler entre autres les noms de Marcel Durliat et de Georges Gaillard, s'occupant surtout de l'art roman du sud de la France et d'Espagne, de René Crozet et, bien sûr, de Louis Grodecki.

C'est lui qui a tenté non seulement une synthèse, la plus aboutie, me semble-t-il, entre le formalisme focillonien et l'examen rigoureux des sources textuelles, mais il a aussi ajouté à ces modèles bien d'autres: Wilhelm Vöge, Adolf Goldschmidt, ou encore Julius von Schlosser. Grodecki publie abondamment sur l'art roman dès les années 1950 et son livre sur L'architecture ottonienne révèle l'étendue de ses intérêts méthodologiques mais aussi son obsession d'une histoire de l'architecture comme typologie structurale. Mais cette ouverture n'a pas grand chose de commun avec celle de Meyer Schapiro: Grodecki n'est pas un "contextualiste » subtil comme Meyer Schapiro. Il convient de noter que dans un article consacré aux chapiteaux romans de l'église de Montermoyen à Bourges (de 1951-52), il salue en Meyer Schapiro un « remarquable connaisseur de la sculpture romane française ». Ce terme de « connaisseur » n'est pas venu par hasard : il dénote combien Grodecki attache plus d'importance à la connaissance du corpus qu'à son analyse contextuelle. Puis il le cite encore dans un article consacré à l'iconographie du tympan de Moissac, et c'est tout. 
Pour les élèves de Grodecki qui ont prolongé ses propres travaux ainsi que ceux de Focillon sur l'art du xI ${ }^{\mathrm{e}}$ siècle, comme Éliane Vergnolle, Noureddine Mezoughi (trop tôt disparu), Maylis Baylé ou Mireille Mentré, les travaux de Meyer Schapiro n'ont d'importance que dans la mesure où ils traitent de la sculpture romane en France ou de points précis relatifs à l'iconographie! La singularité de sa démarche toujours en mouvement n'est guère prise en compte, parce qu'elle est tout simplement étrangère aux deux modalités dominantes que sont le formalisme à la Focillon et l'archéologie façon École des Chartes. Et puis, dans les études publiées en France et vouées à l'art roman, on assiste, dans les années 1990, à un étrange retour sur une forme de « régionalisme » à la Taylor-Nodier... D'ailleurs, on constate que l'art roman a toujours été traité dans notre pays, et cela depuis Arcisse de Caumont, en fonction d' "écoles » (même si le terme est tout de même contesté depuis quelques années), conformément à I'idée que le $\mathrm{XI}^{\mathrm{e}}$ siècle constitue le point de départ d'une sorte de ramification de la " géographie des styles ». On privilégie en France la classification, l'établissement de corpus, avec au mieux un arrière-plan historique, mais en manifestant une grande hostilité envers tout ce qui ressemble à un souci théorique : comme si, sous prétexte qu'elle s'empare d'objets matériels, I'histoire de l'art se faisait sans théorie. Cette absence affichée de théorie est pourtant elle-même une théorie : celle qui, en tout cas, nous éloigne à des années-lumière d'une histoire de l'art à la Meyer Schapiro.

Mais je suis, comment dirais-je, d'un pessimisme nuancé : dans le développement que connaît actuellement l'archéologie du bâti, il me semble que les meilleurs travaux sur l'art roman, ceux qui sauront échapper à une technologie aride, seront caractérisés par ce souci d'une histoire de l'art globale qui animait Meyer Schapiro et dont ses meilleurs articles nous donnent l'exemple. Malheureusement, peu d'enseignants-chercheurs des universités habilités à diriger des recherches sont aujourd'hui attirés par l'étude de l'art roman, même de l'art médiéval en général, alors que notre pays compte un patrimoine monumental exceptionnel pour cette période. II faudra compter sur nos collègues américains ou allemands, italiens ou espagnols...

[Ce débat a été organisé de la manière suivante: les cinq premières questions ont été posées indépendamment aux trois participants ; les deux autres ont été posées aux participants dans l'ordre où leurs réponses apparaissent, chacun pouvant ainsi lire alors la(les) réponse(s) précédente(s). Les trois participants ont naturellement relu l'ensemble du « débat ». (NDLR)]

1. Pour une notice bio-bibliographique, voir le dictionnaire des historiens d'art mis en ligne par la Duke university : www.lib.duke.edu/lilly/

artlibry/dah/schapierom.htm.

2. Voir par exemple le dossier qui lui est consacré dans Journal of Aesthetics and Criticism, 55 :2, Winter, 1997. En outre, deux journées d'études ont été récemment consacrées à la pensée de l'historien : à l'auditorium du Louvre le 28 mai 2005 et à Rome le 24 février 2006.

3. Voir le colloque récemment tenu à Rome, "Meyer Schapiro e i metodi della storia dell'arte », février 2006. Voir Williams, The Art Bulletin, 85 (2003), p. 442-468, et plus particulièrement Michael Camille, « How New York Stole the Idea of Romanesque Art », dans « Medieval, Modern and Postmodern in Meyer Schapiro », dans Oxford Art Journal, 17, 1 (1994), p. 00.
4. "On the Aesthetic Attitude in Romanesque Art", dans Art and Thought: Issued in Honor of Dr. Ananda K. Coomaraswamy on the Occasion of His 70th Birthday, Londres, 1947, p. 130-150. Republié dans Selected Papers (Londres, 1977), Londres, 1993, qui rassemble la plupart des essais de Meyer Schapiro sur l'art roman.

5. "Two Romanesque Drawings in Auxerre and Some Iconographic Problems ", dans Studies in Art and Literature for Belle Da Costa Greene, Princeton, 1954.

6. «A Relief in Rodez and the Beginnings of Romanesque Sculpture in Southern France », dans Millard Meiss éd., Studies in Western Art: Acts of the Twentieth International Congress of the History of Art, vol. I, Princeton, 1963, p. 40-66. Repris dans Romanesque Art, 1977 (1993), p. 285-305. 
7. The Parma Ildefonsus, A Romanesque Illuminated Manuscript from Cluny, and Related Works, New York, 1964.

8. Eliot Norton Lectures, édition en cours de préparation par Linda Seidel.

9. Words and Pictures: On the Literal and Symbolic in the Illustration of a Text, La Haye, 1973, republié dans la collection Words, Scripts and Pictures : Semiotics of Visual Language, New York, 1996, p. 9-113. "On Some Problems in the Semiotics of Visual Arts: Field and Vehicle Image Signs », dans Semiotica 1, 1969, p. 223242 ; repris dans Meyer Schapiro, Theory and Philosophy of Art : Style, Artist and Society, Selected Papers 4, New York, 1994, p. 1-32. Traduction française : «Sur quelques problèmes de sémiotique de l'art visuel : champ et véhicule dans les signes iconiques ", dans Critique, 315-316, 1973, p. 843-866 ; repris également dans Meyer Schapiro, Style, artiste et société, Paris, 1982, p. 7-34. Cet ouvrage est le seul recueil des écrits de Schapiro publié en France; il rassemble plusieurs essais traduits en français. Aucun de ses travaux sur l'art roman n'y figure.

10. The Language of Forms :Lectures On InsularManuscript Art, New York, 2005.

11. "The Apples of Cézanne : An Essay on the Meaning of Still-Life », dans Art News, XXXIV (1968), 34-53, publié en français dans la Revue de l'art, 1-2, 1968, p. 73-87 et repris dans Style, artiste et société, Paris, 1982, p. 171-230.

12. "The Nature of Abstract Art ", dans Marxist Quarterly, 1, 1937, repris dans Modern Art, 19th \& 20th Centuries, Selected Papers 2, New York, 1978, p. 195211 ; publié en français sous le titre « La nature sociale de l'art abstrait », dans Cahiers du Musée national d'art moderne, 4, 1980, p. 271-283, puis dans L'art abstrait, Paris, 1996, p. 9-55.

13. "Leonardo and Freud", dans The Journal of the History of Ideas, XVII, 2, 1956, 147-178, publié en français sous le titre "Deux méprises de Léonard de Vinci suivies d'une erreur de Freud », dans Style, artiste et société, Paris, 1982, p. 139-146.

14. "A Relief... », cité n. 6.

15. Selected Papers, cité n. 4.

16. L'art des sculpteurs romans : recherches sur l'histoire des formes, Paris, 1931.

17. "Review of Kunstwissenschaftliche Forschungen II », dans The Art Bulletin, 18, 2 (1936), p. 258-266.

18. Cité par Walter Cahn, "Schapiro and Focillon", dans Gesta 51, 2, 2002, p. 132 ; les rapports tendus entre les deux hommes sont développés dans une grande partie de l'article.

19. "'Muscipula diaboli': The Symbolism of the Mérode Altarpiece ", The Art Bulletin, 27, 3, (1945), p. 182-187. Traduction française (Daniel Arasse) publiée dans Symboles de la Renaissance, Paris, 1976, p. 39-44.

20. "The Sculptures of Souillac », dans Medieval Studies in Memory of A. Kingsley Porter, tome II, W. R. W. Koehler éd., Cambridge, Mass., 1939, p. 359387, repris dans Romanesque Art, Selected Papers 1, New York, 1977, p. 102-130.

21. "From Mozarabic to Romanesque in Silos ", dans The Art Bulletin, 21 (1939), p. 312-374; repris dans Romanesque Art, Selected Papers 1, New York, 1977, p. 28-101.

22. "A more comprehensive study might lead us to change the conclusions; but it would have to follow the method employed here, the critical correlation of the forms and meanings in the images with historical conditions of the same period and region." (Meyer Schapiro, "From Mozarabic to Romanesque in Silos [1939]", p. 29 (cité n. 21). Voir aussi à cet égard l'article de John Williams, cité n.2. Pour une critique de la pensée marxiste dans les écrits de Schapiro, voir Otto Karl Werckmeister, "Review of Meyer Schapiro's Romanesque Art », dans Art Quarterly 2, (1978), p. 211-18.

23. "The Religious Meaning of the Ruthwell Cross ", dans The Art Bulletin 26, 4 (1944), p. 232-245; repris dans Late Antique, Early Christian and Mediaeval Art, Selected Papers 3, New York, 1979, 150-76.

\section{Cité n. 18.}

25. Conférence donnée au Williamson Alanson White Institute à New York en 1955 et publiée par P. O. Kristeller dans The Journal of History of Ideas, XVII, 1956, 2, p. 147-178. Publiée en français sous le titre "Léonard et Freud : une étude d'histoire de l'art », dans Style, artiste et société, Paris, 1982, p. 93-138.

26. "Style », dans Anthropology Today, A. L. Kroeber éd., Chicago, 1953, p. 287-312 ; repris dans Meyer Schapiro, Theory and Philosophy of Art : Style, Artist, and Society, New York, 1994, 51-102 ; publié en français sous le titre " La notion de style », dans Style, artiste et société Paris, 1982, p. 35-86.

27. L'arte moderna, Turin, 1988.

28. Giovanna Perini, M. Schapiro, Per una semiotica del linguaggio visivo, Rome, 2002.

29. Storia della critica d'arte, Turin, 1936.

30. Proposte per una critica d'arte, Florence, 1950.

31. Michael Camille, "How New York Stole the Idea of Romanesque Art », dans Oxford Art Journal, 17, 1, 1994, p. 65-75.

32. Ces sujets sont au cœur des contributions de plusieurs spécialistes à paraître l'année prochaine dans R. Maxwell, K. Ambrose éd., New Directions in Romanesque Sculpture Studies.

33. Walter Cahn, Linda Seidel, Romanesque Sculpture in American Collections, vol. 1, New York, 1979. 